Internat. J. Math. \& Math. Sci.

Vol. 22, No. 3 (1999) 469-482

S 0161-1712<99>22469-6

(c) Electronic Publishing House

\title{
SIX-DIMENSIONAL CONSIDERATIONS OF EINSTEIN'S CONNECTION FOR THE FIRST TWO CLASSES I. THE RECURRENCE RELATIONS IN 6-g-UFT
}

\author{
KYUNG TAE CHUNG, GYE TAK YANG, and IN HO HWANG
}

(Received 31 July 1998)

\begin{abstract}
Lower dimensional cases of Einstein's connection were already investigated by many authors for $n=2,3,4,5$. This paper is the first part of the following series of two papers, in which we obtain a surveyable tensorial representation of 6-dimensional Einstein's connection in terms of the unified field tensor, with main emphasis on the derivation of powerful and useful recurrence relations which hold in 6-dimensional Einstein's unified field theory (i.e., 6-g-UFT):

I. The recurrence relations in 6-g-UFT.

II. The Einstein's connection in 6-g-UFT.

All considerations in these papers are restricted to the first and second classes only, since the case of the third class, the simplest case, was already studied by many authors.

Keywords and phrases. Generalized Riemannian manifold, 6-dimensional Einstein's unified field theory, recurrence relations in $6-g$-UFT.
\end{abstract}

1991 Mathematics Subject Classification. 83E50, 83C05, 58A05.

1. Introduction. In Appendix II to his last book, Einstein ([12], 1950) proposed a new unified field theory that would include both gravitation and electromagnetism. Although the intent of this theory is physical, its exposition is mainly geometrical. It may be characterized as a set of geometrical postulates for the space-time $X_{4}$. Characterizing Einstein's unified field theory as a set of geometrical postulates in $X_{4}$, Hlavatý ([13], 1957) gave its mathematical foundation for the first time. Since then Hlavatý and number of mathematicians contributed to the development of this theory and obtained many geometrical consequences of these postulates.

Generalizing $X_{4}$ to $n$-dimensional generalized Riemannian manifold $X_{n}, n$-dimensional generalization of this theory, so called Einstein's $n$-dimensional unified field theory ( $n$-g-UFT hereafter), had been attempted by Wrede ([16], 1958) and Mishra ([15], 1959). On the other hand, corresponding to $n$-g-UFT, Chung ([1], 1963) introduced a new unified field theory, called the Einstein's $n$-dimensional ${ }^{*} g$-unified field theory $(n$ ${ }^{*} g$-UFT hereafter). This theory is more useful than $n$ - $g$-UFT in some physical aspects. Chung and et al. obtained many results concerning this theory ([2], 1969; [7], 1981; [8], 1988; [5, 6], 1998), particularly proving that $n$ - $^{*} \mathrm{~g}$-UFT is equivalent to $n-g$-UFT so far as the classes and indices of inertia are concerned ([3], 1985). The case of the third class, which is the simplest case of both unified field theories, was completely studied for a general $n$ by many authors $([15,16,8]$, etc.) However, in the cases of the first and second classes of both $n$-dimensional generalizations, it has been unable yet to 
represent the general $n$-dimensional Einstein's connection in a surveyable tensorial form in terms of the unified field tensor $g_{\lambda \mu}$. This is probably due to the complexity of the higher dimensions.

However, the lower dimensional cases of the Einstein's connection in $n$-g-UFT were investigated by many authors: 2-dimensional case by Jakubowicz ([14], 1969) and Chung et al. ([4], 1983), 3-dimensional case by Chung et al. ([10, 11, 9], 1979-1981), and 4 -dimensional case by Hlavatý ([13], 1957) and many other geometers. Recently, Chung et al. also studied the Einstein's connection in $4{ }^{*} g$-UFT $([1], 1963)$ and $3-$ and 5 -* $g$-UFT ([8], 1988), and obtained respective Einstein's connection in a surveyable tensorial form.

The purpose of the present paper, the first part of a series of two papers, is to derive powerful recurrence relations which hold in 6 - $g$-UFT. In the second part, we prove a necessary and sufficient condition for the existence and uniqueness of the Einstein's connection in 6-g-UFT and establish a linear system of 43 equations for the solution of 6-dimensional Einstein's connection, employing the powerful recurrence relations obtained in Part I.

All considerations in this and subsequent papers are dealt with for the first and second classes only.

2. Preliminaries. This section is a brief collection of basic concepts, notations, and results, which are needed in our subsequent considerations. They are due to Chung ([1], 1963; [8], 1988), Hlavatý ([13], 1957) and Mishra ([15], 1959). All considerations in this section are dealt with for a general $n>1$.

2.1. $n$-dimensional $g$-unified field theory. The Einstein's $n$-dimensional unified field theory, denoted by $n-g$-UFT, is an $n$-dimensional generalization of the usual Einstein's 4-dimensional unified field theory in the space-time $X_{4}$. It is based on the following three principles as indicated by Hlavatý [13].

Principle A. Let $X_{n}$ be an $n$-dimensional generalized Riemannian manifold referred to a real coordinate system $x^{v}$, which obeys the coordinate transformation $x^{v} \rightarrow x^{v^{\prime}}$. (Throughout the present paper, Greek indices are used for the holonomic components of tensors, while Roman indices are used for the nonholonomic components of a tensor in $X_{n}$. All indices take the values $1,2, \ldots, n$, and follow the summation convention with the exception of nonholonomic indices $x, y, z, t$.) for which

$$
\operatorname{det}\left(\frac{\partial x^{\prime}}{\partial x}\right) \neq 0
$$

In $n$ - $g$-UFT the manifold $X_{n}$ is endowed with a real nonsymmetric tensor $g_{\lambda \mu}$, called the unified field tensor of $X_{n}$. This tensor may be decomposed into its symmetric part $h_{\lambda \mu}$ and skew-symmetric part $k_{\lambda \mu}$

$$
g_{\lambda \mu}=h_{\lambda \mu}+k_{\lambda \mu},
$$

where

$$
\mathfrak{g}=\operatorname{det}\left(g_{\lambda \mu}\right) \neq 0, \quad \mathfrak{h}=\operatorname{det}\left(h_{\lambda \mu}\right) \neq 0, \quad \mathfrak{k}=\operatorname{det}\left(k_{\lambda \mu}\right) .
$$


We may define a unique tensor $h^{\lambda v}=h^{v \lambda}$ by

$$
h_{\lambda \mu} h^{\lambda v}=\delta_{\mu}^{v}
$$

In $n$ - $g$-UFT the tensors $h_{\lambda \mu}$ and $h^{\lambda v}$ will serve for raising and/or lowering indices of tensors in $X_{n}$ in the usual manner.

Principle B. The differential geometric structure on $X_{n}$ is imposed by the tensor $g_{\lambda \mu}$ by means of a connection $\Gamma_{\lambda \mu}^{\nu}$ defined by a system of equations

$$
D_{\omega} g_{\lambda \mu}=2 S_{\omega \mu}^{\alpha} g_{\lambda \alpha}
$$

Here $D_{\omega}$ denotes the symbolic vector of the covariant derivative with respect to $\Gamma_{\lambda \mu}^{v}$ and $S_{\lambda \mu}{ }^{\nu}$ is the torsion tensor of $\Gamma_{\lambda \mu}^{v}$. The connection $\Gamma_{\lambda \mu}^{v}$ satisfying (2.4) is called the Einstein's connection. Under certain conditions the system (2.4) admits a unique solution $\Gamma_{\lambda \mu}^{\nu}$.

PRINCIPLE C. In order to obtain $g_{\lambda \mu}$ involved in the solution for $\Gamma_{\lambda \mu}^{\nu}$ certain conditions are imposed. These conditions may be condensed to

$$
S_{\lambda}=S_{\lambda \alpha}{ }^{\alpha}=0, \quad R_{[\mu \lambda]}=\partial_{[\mu} X_{\lambda]},
$$

where $X_{\lambda}$ is an arbitrary nonzero vector, and $R_{\omega \mu \lambda}{ }^{v}$ and $R_{\mu \lambda}$ are the curvature tensors of $\Gamma_{\lambda \mu}^{v}$ defined by

$$
R_{\omega \mu \lambda}{ }^{v}=2\left(\partial_{[\mu} \Gamma_{|\lambda| \omega]}^{v}+\Gamma_{\alpha[\mu}^{v} \Gamma_{|\lambda| \omega]}^{\alpha}\right), \quad R_{\mu \lambda}=R_{\alpha \mu \lambda}^{\alpha} .
$$

2.2. Algebraic preliminaries. In this subsection, notations, concepts, and several algebraic results in $n$ - $g$-UFT are introduced.

(i) NotAtions. The following scalars, tensors, and notations are frequently used in our further considerations.

$$
\begin{gathered}
g=\frac{\mathfrak{g}}{\mathfrak{h}}, \quad k=\frac{\mathfrak{k}}{\mathfrak{h}}, \\
K_{p}=k_{\left[\alpha_{1}\right.}{ }^{\alpha_{1}} k_{\alpha_{2}}{ }^{\alpha_{2}} \cdots k_{\left.\alpha_{p}\right]}{ }^{\alpha_{p}}, \quad(p=0,1,2, \ldots), \\
{ }^{(0)} k_{\lambda}{ }^{v}=\delta_{\lambda}^{v}, \quad{ }^{(1)} k_{\lambda}{ }^{v}=k_{\lambda}{ }^{v}, \quad{ }^{(p)} k_{\lambda}{ }^{v}={ }^{(p-1)} k_{\lambda}{ }^{\alpha} k_{\alpha}{ }^{v}, \quad(p=1,2, \ldots), \\
K_{\omega \mu \nu}=\nabla_{\nu} k_{\omega \mu}+\nabla_{\omega} k_{v \mu}+\nabla_{\mu} k_{\omega \nu}, \\
\sigma= \begin{cases}0, & \text { if } n \text { is even, } \\
1, & \text { if } n \text { is odd, }\end{cases}
\end{gathered}
$$

where $\nabla_{\omega}$ is the symbolic vector of covariant derivative with respect to the Christoffel 
symbols $\left\{\begin{array}{c}v \\ \lambda \mu\end{array}\right\}$ defined by $h_{\lambda \mu}$. It has been shown that the scalars and tensors introduced in (2.7) satisfy

$$
\begin{gathered}
K_{0}=1, \quad K_{n}=k \quad \text { if } n \text { is even and } \quad K_{p}=0 \text { if } p \text { is odd, } \\
g=1+K_{2}+\cdots+K_{n-\sigma}, \\
{ }^{(p)} k_{\lambda \mu}=(-1)^{P(p)} k_{\mu \lambda}, \quad{ }^{(p)} k^{\lambda \nu}=(-1)^{P(p)} k^{v \lambda .}
\end{gathered}
$$

Furthermore, we also use the following useful abbreviations, denoting an arbitrary tensor $T_{\omega \mu \lambda}$, skew-symmetric in the first two indices, by $T$ :

$$
\begin{aligned}
& \stackrel{p q r}{T}=\stackrel{p q r}{T}{ }_{\omega \mu \lambda}=T_{\alpha \beta \gamma}{ }^{(p)} k_{\omega}{ }^{\alpha(q)} k_{\mu}{ }^{\beta(r)} k_{\lambda}^{\gamma}, \\
& T=T_{\omega \mu \lambda}=\stackrel{000}{T}, \\
& 2 \stackrel{p q r}{T}_{\omega[\lambda \mu]}=\stackrel{p q r}{T_{\omega \lambda \mu}-{ }^{T} T_{\omega \mu \lambda},} \quad 2 \stackrel{(p q) r}{T} T_{\omega \lambda \mu}=\stackrel{p q r}{T_{\omega \lambda \mu}}+\stackrel{q p r}{T} T_{\omega \lambda \mu} \text {, etc. }
\end{aligned}
$$

We then have

$$
\stackrel{p q r}{T_{\omega \lambda \mu}}=-\stackrel{q p r}{T_{\lambda \omega \mu}}
$$

(ii) ClassificATION, BASIC VECTORS, AND BASIC SCALARS

DEFINITION 2.1. The tensor $g_{\lambda \mu}$ (or $k_{\lambda \mu}$ ) is said to be

(1) of the first class, if $K_{n-\sigma} \neq 0$;

(2) of the second class with the $j$ th category $(j \geq 1)$, if

$$
K_{2 j} \neq 0, \quad K_{2 j+2}=K_{2 j+4}=\cdots=K_{n-\sigma}=0 ;
$$

(3) of the third class, if $K_{2}=K_{4}=\cdots=K_{n-\sigma}=0$.

The solution of the system of equations (2.4) is most conveniently brought about in a nonholonomic frame of reference, which may be introduced by the projectivity

$$
M A^{v}=k_{\mu}{ }^{v} A^{\mu}, \quad(M \text { a scalar }) .
$$

DEFINITION 2.2. An eigenvector $A^{v}$ of $k_{\lambda \mu}$ that satisfies (2.12) is called a basic vector in $X_{n}$, and the corresponding eigenvalue $M$ is termed a basic scalar.

It has been shown that the basic scalars $M$ are solutions of the characteristic equation

$$
M^{\sigma}\left(M^{n-\sigma}+K_{2} M^{n-2-\sigma}+\cdots+K_{n-2-\sigma} M^{2}+K_{n-\sigma}\right)=0 .
$$

(iii) NONHOLONOMIC FRAME OF REFERENCE. In the first and second classes, we have a set of $n$ linearly independent basic vectors ${\underset{i}{i}}_{i}^{v}(i=1, \ldots, n)$ and a unique reciprocal set $\stackrel{i}{A_{\lambda}}(i=1, \ldots, n)$, satisfying

$$
\stackrel{j}{A_{\lambda}} \underset{i}{A^{\lambda}}=\delta_{i}^{j}, \quad \stackrel{i}{A_{\lambda}} \underset{i}{A^{v}}=\delta_{\lambda}^{v} .
$$

With these two sets of vectors, we may construct a nonholonomic frame of reference as follows: 
DEFINITION 2.3. If $T_{\lambda \cdots}^{v \cdots}$ are holonomic components of a tensor, then its nonholonomic components $T_{j \ldots}^{i \cdots}$ are defined by

$$
T_{j \cdots}^{i \cdots}=T_{\lambda \cdots}^{\nu \cdots} \stackrel{i}{A}_{\nu} \cdots \underset{j}{A_{j}^{\lambda} \cdots .}
$$

An easy inspection shows that

$$
T_{\lambda \cdots}^{v \cdots}=T_{j \cdots}^{i \cdots} A_{i}^{v} \cdots \stackrel{j}{A}_{\lambda} \cdots .
$$

Furthermore, if $\underset{x}{M}$ is the basic scalar corresponding to $\underset{x}{\underset{x}{v}}$, then the nonholonomic components of ${ }^{(p)} k_{\lambda}{ }^{v}$ are given by

$$
{ }^{(p)} k_{x}{ }^{i}=M_{x}^{p} \delta_{x}^{i}, \quad{ }^{(p)} k_{x i}={\underset{x}{M}}^{p} h_{x i}, \quad{ }^{(p)} k^{x i}={\underset{x}{M}}^{p} h^{x i} .
$$

Without loss of generality we may choose the nonholonomic components of $h_{\lambda \mu}$ as

$$
\begin{aligned}
h_{12} & =h_{34}=\cdots=h_{n-1-\sigma, n-\sigma}=1, \\
\sigma h_{n i_{0}} & =\delta_{\sigma}^{1}, \quad \text { remaining } h_{i j}=0,
\end{aligned}
$$

where the index $i_{0}$ is taken so that $\operatorname{det}\left(h_{i j}\right) \neq 0$ when $n$ is odd.

2.3. Differential geometric preliminaries. In this subsection, we present several useful results involving Einstein's connection. These results are needed in our subsequent considerations for the solution of (2.4).

If the system (2.4) admits a solution $\Gamma_{\lambda \mu}^{v}$, it must be of the form

$$
\Gamma_{\lambda \mu}^{v}=\left\{\begin{array}{c}
v \\
\lambda \mu
\end{array}\right\}+S_{\lambda \mu}^{v}+U_{\lambda \mu}^{v},
$$

where

$$
U_{v \lambda \mu}=2 \stackrel{001}{S} v(\lambda \mu) \text {. }
$$

The above two relations show that our problem of determining $\Gamma_{\lambda \mu}^{\nu}$ in terms of $g_{\lambda \mu}$ is reduced to that of studying the tensor $S_{\lambda \mu}{ }^{\nu}$. On the other hand, it has been shown that the tensor $S_{\lambda \mu}{ }^{v}$ satisfies

$$
S=B-3 \stackrel{(110)}{S}
$$

where

$$
2 B_{\omega \mu \nu}=K_{\omega \mu \nu}+3 K_{[\alpha \beta \nu]} k_{\omega}{ }^{\alpha} k_{\mu}{ }^{\beta} .
$$

Therefore, the Einstein's connection $\Gamma_{\lambda \mu}^{\nu}$ satisfying (2.4) may be determined if the solution $S_{\lambda \mu}{ }^{v}$ of the system (2.20) is found. The main purpose of the present paper is to find a device to solve the system (2.20) when $n=6$.

Furthermore, for the first two classes, the nonholonomic solution of (2.20) is given by

$$
\underset{x y z}{M} S_{x y z}=B_{x y z}
$$

or equivalently

$$
2 \underset{x y z}{M} S_{x y z}=K_{x y z}+3 K_{[x y z]} \underset{x}{M} \underset{y}{M},
$$

where

$$
\underset{x y z}{M}=1+\underset{x}{M} M_{y}+\underset{y}{M} \underset{z}{M}+\underset{z}{M}
$$


Therefore, in virtue of (2.22), we see that a necessary and sufficient nonholonomic conditions for the system (2.4) to have a unique solution in the first two classes is

$$
\underset{x y z}{M} \neq 0 \text { for all } x, y, z \text {. }
$$

3. The recurrence relations of the first kind in $n$-g-UFT. This section is devoted to the derivation of the recurrence relations of the first kind and two other useful relations which hold in $n$-g-UFT. All considerations in this section are also dealt with for a general $n>1$.

The recurrence relations of the first kind in $n-g$-UFT are those which are satisfied by the tensors ${ }^{(p)} k_{\lambda}{ }^{v}$. These relations will be proved in the following theorem.

THEOREM 3.1 (The recurrence relations of the first kind in $n-g$-UFT). The tensors ${ }^{(p)} K_{\lambda}{ }^{v}$ satisfy the following recurrence relations:

FOR THE FIRST CLASS.

$$
{ }^{(n+p)} k_{\lambda}{ }^{v}+K_{2}{ }^{(n+p-2)}{k_{\lambda}}^{v}+\cdots+K_{n-\sigma-2}{ }^{(p+\sigma+2)}{k_{\lambda}}^{v}+K_{n-\sigma}{ }^{(p+\sigma)} k_{\lambda}{ }^{v}=0
$$

which may be condensed to

$$
\sum_{f=0}^{n-\sigma} K_{f}^{(n+p-f)} k_{\lambda}^{v}=0, \quad(p=0,1,2, \ldots) .
$$

FOR THE SECOND CLASS WITH THE $j$ th CATEGORY.

$$
{ }^{(2 j+p)}{k_{\lambda}}^{v}+K_{2}{ }^{(2 j+p-2)} k_{\lambda}{ }^{v}+\cdots+K_{2 j}{ }^{(p)} k_{\lambda}{ }^{v}=0
$$

which may be condensed to

$$
\sum_{f=0}^{2 j} K_{f}^{(2 j+p-f)} k_{\lambda}^{v}=0, \quad(p=1,2, \ldots) .
$$

PROOF OF THE CASE OF THE FIRST ClASS. Let $\underset{x}{M}$ be a basic scalar. Then, in virtue of (2.13), we have

$$
\sum_{f=0}^{n-\sigma} K_{f} \underset{x}{M^{n-f}}=0
$$

Multiplying $\delta_{x}^{i}$ to both sides of (3.3) and making use of (2.16), we have

$$
\sum_{f=0}^{n-\sigma} K_{f}^{(n-f)} k_{x}^{i}=0
$$

whose holonomic form is

$$
\sum_{f=0}^{n-\sigma} K_{f}^{(n-f)} k_{\lambda}{ }^{\alpha}=0 .
$$

The relation (3.1) immediately follows by multiplying ${ }^{(P)} k_{\alpha}{ }^{v}$ to both sides of (3.4b).

Proof OF THE CASE OF THE SECOND ClASS WITH THE $j$ TH CATEgory. When $g_{\lambda} \mu$ belongs to the second class with the $j$ th category, the characteristic equation (2.13) is reduced to 


$$
\sum_{f=0}^{2 j} K_{f} M^{n-f}=M^{n-2 j} \sum_{f=0}^{2 j} K_{f} M^{2 j-f}=0 .
$$

Hence, if $\underset{x}{M}$ is a root of (3.5a), it satisfies

$$
\underset{x}{M} \sum_{f=0}^{2 j} K_{f} \underset{x}{M^{2 j-f}}=\sum_{f=0}^{2 j} K_{f} \underset{x}{M^{2 j-f+1}}=0 .
$$

In virtue of (2.16), multiplication of $\delta_{x}^{i}$ to both sides of (3.5b) gives

$$
\sum_{f=0}^{2 j} K_{f}^{(2 j-f+1)} k_{x}^{i}=0 .
$$

The holonomic form of (3.6a) is

$$
\sum_{f=0}^{2 j} K_{f}^{(2 j-f+1)} k_{\lambda}{ }^{\alpha}=0 .
$$

Consequently, the relation (3.2) follows by multiplying ${ }^{(p-1)} k_{\alpha}{ }^{v}$ to both sides of (3.6b).

REMARK 3.2. When $g_{\lambda \mu}$ belongs to the second class with the first category, the relation (3.2) is reduced to

$$
{ }^{(p+2)} k_{\lambda}{ }^{v}+K_{2}{ }^{(p)} k_{\lambda}{ }^{v}=0, \quad(p=1,2, \ldots) .
$$

In the following two theorems we prove two useful relations.

THEOREM 3.3 (For the first and second classes). In the first two classes, a tensor $T_{\omega \mu \nu}$, skew-symmetric in the first two indices, satisfies

$$
\begin{aligned}
& \stackrel{(p q) r}{T}_{\omega \mu \nu}=\sum_{x, y, z} T_{x y z}{\underset{x}{M}}_{(p}^{M_{y}^{q q)}} \underset{z}{M^{r}} \stackrel{x}{A_{\omega}} \underset{A_{\mu} A_{v}}{y},
\end{aligned}
$$

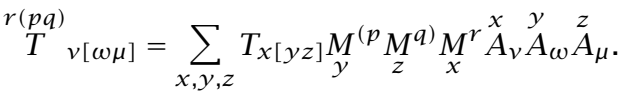

Proof. Making use of (2.15b) and (2.17), the relation (3.8a) may be proved as in the following way:

$$
\begin{aligned}
& \stackrel{(p q) r}{T}_{\omega \mu \nu}=\sum_{x, y, z} \stackrel{(p q) r}{T}_{x y z} \underset{A_{\omega}}{x} \underset{A_{\mu}}{y} A_{\nu} \\
& =\frac{1}{2} \sum_{x, y, z} T_{i j k}\left[{ }^{(p)} k_{x}{ }^{i(q)} k_{y}{ }^{j}+{ }^{(q)} k_{x}{ }^{i(p)} k_{y}\right]^{j(r)} k_{z}{ }^{k} \stackrel{x}{A_{\omega}} \underset{A_{\mu}}{\quad z} A_{v} \\
& =\frac{1}{2} \sum_{x, y, z} T_{x y z}\left(\underset{x}{M^{p}} M_{y}^{q}+\underset{x}{M^{q}} M_{y}^{p}\right) \underset{z}{M^{r}} \stackrel{x}{A_{\omega}} \underset{A_{\mu}}{y} A_{\nu} .
\end{aligned}
$$

The second relation can be proved similarly. 
THEOREM 3.4 (For all cases). The tensor $B_{\omega \mu \nu}$, given by (2.21), satisfies

$$
\begin{aligned}
& \stackrel{(p q) r}{B}=\stackrel{(p q) r}{S}+\stackrel{\left(p^{\prime} q^{\prime}\right) r}{S}+\stackrel{\left(p^{\prime} q\right) r^{\prime}}{S}+\stackrel{\left(p q^{\prime}\right) r^{\prime}}{S} \\
& 2 \stackrel{(p q) r}{B} \omega \mu \nu=\stackrel{(p q) r}{K} \omega \mu \nu+\stackrel{\left(p^{\prime} q^{\prime}\right) r}{K} \omega \mu \nu+\stackrel{\left(p^{\prime} q\right) r^{\prime}}{K}{ }_{v[\omega \mu]}+\stackrel{\left(p q^{\prime}\right) r^{\prime}}{K} \quad v[\omega \mu],
\end{aligned}
$$

where

$$
p^{\prime}=p+1, \quad q^{\prime}=q+1, \quad r^{\prime}=r+1 .
$$

Proof. In virtue of (2.9) and (2.20), the relation (3.10) may be shown as in the following way:

$$
\begin{aligned}
\stackrel{(p q) r}{B}=^{(}{ }_{B}^{(p q) r} & =\frac{1}{2} B_{\alpha \beta \gamma}\left[{ }^{(p)} k_{\omega}{ }^{\alpha(q)} k_{\mu}{ }^{\beta}+{ }^{(q)} k_{\omega}{ }^{\alpha(p)} K_{\mu}{ }^{\beta}\right]^{(r)} k_{\nu}{ }^{\gamma} \\
= & \frac{1}{2}\left[S_{\alpha \beta \gamma}+S_{\epsilon \eta \gamma} k_{\alpha}{ }^{\epsilon} k_{\beta}{ }^{\eta}+S_{\epsilon \beta \eta} k_{\alpha}{ }^{\epsilon} k_{\gamma}{ }^{\eta}+S_{\alpha \epsilon \eta} k_{\beta}{ }^{\epsilon} k_{\gamma}{ }^{\eta}\right] \\
& \times\left[{ }^{(p)} k_{\omega}{ }^{\alpha(q)} k_{\mu}{ }^{\beta}+{ }^{(q)} k_{\omega}{ }^{\alpha(p)} k_{\mu}{ }^{\beta}\right]^{(r)} k_{\nu}{ }^{\gamma} .
\end{aligned}
$$

After a lengthy calculation, we note that the right-hand side of the above equation is equal to (3.10). The relation (3.11) may be proved similarly.

4. The recurrence relations of the second and third kinds in $6-g$-UFT. This section is specially concerned with the 6-dimensional case; that is with 6 - $g$-UFT. In this section, we first investigate the basic scalars and some relations satisfied by them. In order to obtain a tensorial representation of the 6-dimensional Einstein's connection $\Gamma_{\lambda \mu}^{v}$ in terms of $g_{\lambda \mu}$, we need powerful recurrence relations of the third kind which are satisfied by an arbitrary tensor $T_{\omega \lambda \mu}$, skew-symmetric in the first two indices. Therefore, we finally derive these relations, after introducing the recurrence relations of the second kind which are satisfied by the basic scalars. All considerations in this section are restricted to $n=6$.

In 6- $g$-UFT there are four cases; that is, the unified field tensor $g_{\lambda \mu}$ belongs to

(1) the first class, if $K_{6} \neq 0$,

(2) the second class with the first category, if $K_{2} \neq 0, K_{4}=K_{6}=0$,

(3) the second class with the second category, if $K_{4} \neq 0, K_{6}=0$,

(4) the third class, if $K_{2}=K_{4}=K_{6}=0$.

In this section we investigate the first three cases.

Before we start investigations about the basic scalars, we first note that in 6 - $g$-UFT the relation $(2.8 \mathrm{~b})$ is reduced to

$$
g=1+K_{2}+K_{4}+K_{6}
$$

and formally state in the following theorem the recurrence relations of the first kind when $n=6$, which are direct consequences of (3.1) and (3.2). 
THEOREM 4.1 (The recurrence relations of the first kind in 6-g-UFT). The tensors ${ }^{(p)} k_{\lambda}{ }^{v}$ satisfy the following recurrence relations in $6-g$-UFT for $p=0,1,2, \ldots$ :

\begin{tabular}{|c|c|c|}
\hline Class and Category & Recurrence relations of the first kind in $6-g-U F$ & \\
\hline The first class & ${ }^{(p+6)} k_{\lambda}{ }^{v}=-K_{2}{ }^{(p+4)} k_{\lambda}{ }^{v}-K_{4}{ }^{(p+2)} k_{\lambda}{ }^{v}-K_{6}{ }^{(p)} k_{\lambda}{ }^{v}$ & $(4.2 \mathrm{a})$ \\
\hline $\begin{array}{l}\text { The second class with } \\
\text { the second category }\end{array}$ & ${ }^{(p+4)} k_{\lambda}{ }^{v}=-K_{2}{ }^{(p+2)} k_{\lambda}{ }^{v}-K_{4}{ }^{(p)} k_{\lambda}{ }^{v}$. & $(4.2 \mathrm{~b})$ \\
\hline $\begin{array}{l}\text { The second class with } \\
\text { the first category }\end{array}$ & ${ }^{(p+2)} k_{\lambda}{ }^{v}=-K_{2}{ }^{(p)} k_{\lambda}{ }^{v}$. & $(4.2 \mathrm{c})$ \\
\hline
\end{tabular}

THEOREM 4.2. The basic scalars in 6-g-UFT are given by

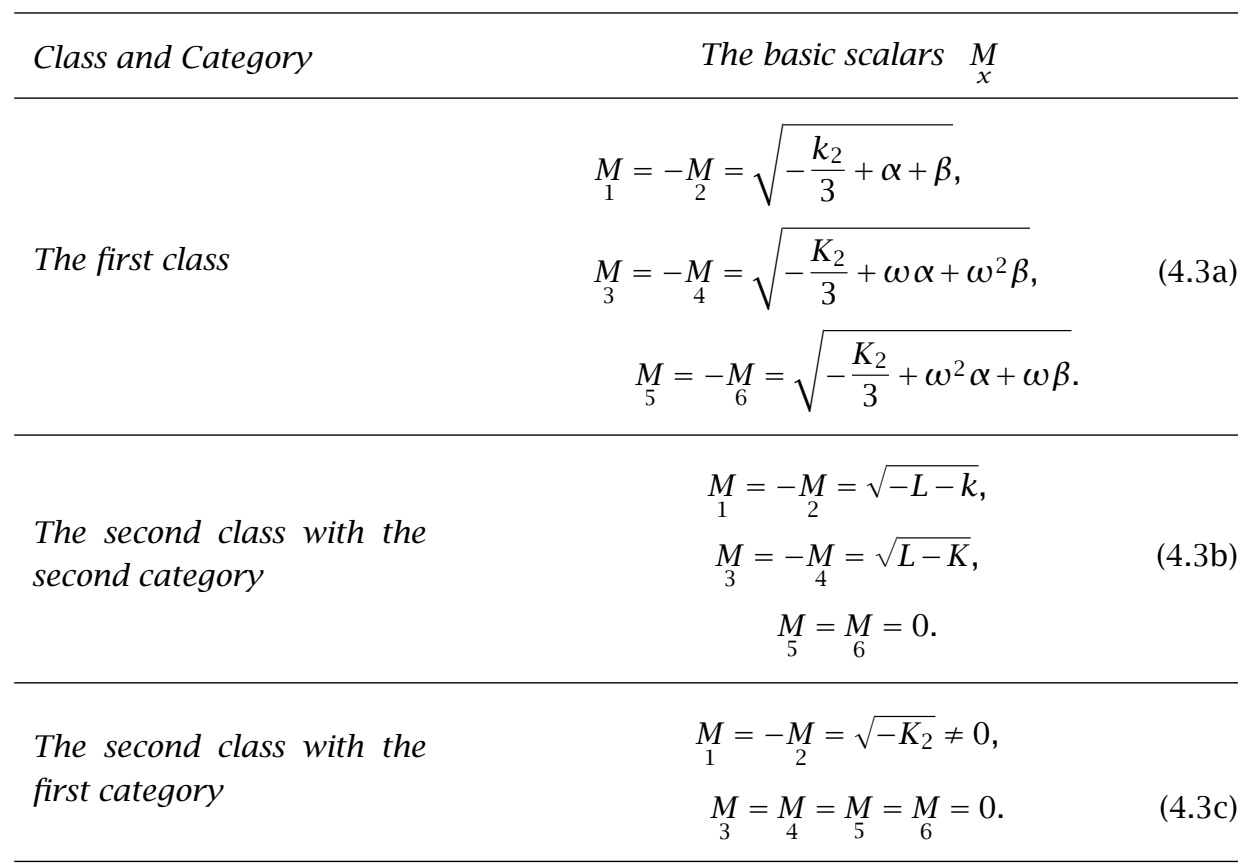

where

$$
\begin{gathered}
\omega=\frac{-1+\sqrt{3} i}{2}, \\
\alpha=\left[-\frac{\phi}{2}+\sqrt{\left(\frac{\phi}{2}\right)^{2}+\left(\frac{\theta}{3}\right)^{3}}\right]^{1 / 3},
\end{gathered}
$$




$$
\begin{aligned}
\beta & =\left[-\frac{\phi}{2}-\sqrt{\left(\frac{\phi}{2}\right)^{2}+\left(\frac{\theta}{3}\right)^{3}}\right]^{1 / 3}, \\
\theta & =K_{4}-\frac{\left(K_{2}\right)^{2}}{3}, \\
\phi & =K_{6}-\frac{K_{2} K_{4}}{3}+\frac{2}{27}\left(K_{2}\right)^{3}, \\
L & =\sqrt{\left(\frac{K_{2}}{2}\right)^{2}-K_{4}}, \quad K=\frac{K_{2}}{2} .
\end{aligned}
$$

Proof. Since the characteristic equation (2.13) for the first class in 6 - $g$-UFT is reduced to

$$
\left(M^{2}\right)^{3}+K_{2}\left(M^{2}\right)^{2}+K_{4} M^{2}+K_{6}=0
$$

equation (4.3a) follows by the method of Cardano, using the notations given by (4.4a). In this case we note that all basic scalars are not zero in virtue of (4.6d). The other cases may be shown similarly.

THEOREM 4.3. The basic scalars $\underset{x}{M}$ in 6-g-UFT satisfy the following relations:

The first class

$$
\begin{gathered}
\underset{1}{M}+\underset{2}{M}=\underset{3}{M}+\underset{4}{M}=\underset{5}{M}+\underset{6}{M}=0, \\
\underset{a}{M}+\underset{b}{M^{2}}+\underset{c}{M^{2}}=-K_{2}, \\
\underset{a}{M_{b}^{2}} \underset{b}{M^{2}}+\underset{c}{M^{2}} M_{c}^{2}+\underset{c}{M_{a}^{2}} \underset{a}{M^{2}}=K_{4}, \\
\underset{a}{M_{b}^{2}} \underset{c}{M^{2}} M^{2}=-K_{6} .
\end{gathered}
$$

The second class with the

$$
\begin{gathered}
\underset{1}{M}+\underset{2}{M}=\underset{3}{M}+\underset{4}{M}=\underset{5}{M}+\underset{6}{M}=0, \\
\underset{a}{M} \underset{c}{M}=\underset{b}{M} \underset{c}{M}=0, \quad \underset{a}{M}+\underset{b}{M}=-K_{2}, \\
\underset{a}{M} M_{b}^{M^{2}}=K_{4}, \quad \underset{a}{M^{2}} M_{c}^{2}=\underset{b}{M_{c}^{2}} M^{2}=0 .
\end{gathered}
$$

The second class with the first category $(x, y=b, c)$

$$
\begin{gathered}
\underset{1}{M+\underset{2}{M}}=\underset{x}{M}+\underset{y}{M}=0, \\
\underset{12}{M}=K_{2}, \quad \underset{1}{M} \underset{x}{M}=\underset{2}{M} \underset{x}{M}=\underset{x}{M} \underset{y}{M}=0 .
\end{gathered}
$$

Here, the indices $a, b, c$ are assumed to take values as $a=1,2 ; b=3,4 ; c=5,6$. 
Proof. The relations (4.6), (4.7), and (4.8) follow from (4.3), (4.3b), and (4.3c), respectively. In the proof of (4.6), use of the following relations are made

$$
\begin{aligned}
\omega^{2} & =-1-\omega, \quad \omega^{3}=1, \\
\alpha \beta & =-\frac{1}{3} K_{4}+\frac{1}{9}\left(K_{2}\right)^{2}, \\
\alpha^{3}+\beta^{3} & =-K_{6}+\frac{K_{2} K_{4}}{3}-\frac{2}{27}\left(K_{2}\right)^{3} .
\end{aligned}
$$

Using the relations given in Theorem 4.3, we may prove the recurrence relations of the second kind in the following theorem.

THEOREM 4.4 (The recurrence relations of the second kind in 6-g-UFT). In 6-g-UFT the basic scalars $M_{x}$ satisfy the following recurrence relations which hold for all values of $x$ and $y$ when $x \neq y$ :

FOR THE FIRST CLASS.

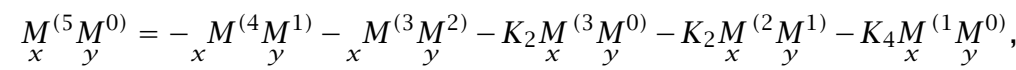

$$
\begin{aligned}
& 2 \underset{x}{2}{ }^{(5}{ }_{y}^{1)}=-\underset{x}{M}{ }_{y}^{3}{ }^{3}-2 K_{2} \underset{x}{M}{ }_{y}^{(3} M^{1)}-K_{4} \underset{x}{M} \underset{y}{M} \\
& \underset{x}{M}{ }_{y}^{(5} M^{2)}=-\underset{x}{M}{ }_{y}^{(4}{\underset{y}{3)}}^{M_{2}} \underset{x}{M^{(3}} \underset{y}{M^{2)}}+K_{6} \underset{x}{M^{(1}}{\underset{y}{M}}^{0)}, \\
& 2 \underset{x}{M^{(5}} \underset{y}{M^{3)}}=-\underset{x}{M^{4}} \underset{y}{M^{4}}-K_{2} \underset{x}{M^{3}} \underset{y}{M^{3}}+K_{4} \underset{x}{M^{2}} \underset{y}{M^{2}}+2 K_{6} \underset{x}{M^{(2}} \underset{y}{M^{0)}}+K_{6} \underset{x}{M} \underset{y}{M} \text {, }
\end{aligned}
$$

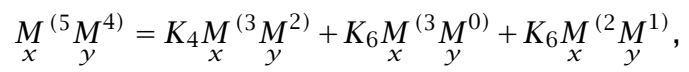

$$
\begin{aligned}
& \underset{x}{M^{5}} \underset{y}{M^{5}}=K_{2} \underset{x}{M_{y}^{4}} \underset{y}{M^{4}}+2 K_{4} \underset{x}{M_{y}^{(4}} \underset{y}{M^{2)}}+2 K_{6} \underset{x}{M_{y}^{(4}}{\underset{y}{0)}}^{0)} \\
& +K_{4} \underset{x}{M^{3}} \underset{y}{M^{3}}+2 K_{6} \underset{x}{M_{y}^{(3}} M^{1)}+K_{6} \underset{x}{M_{y}^{2}} \underset{y}{M^{2}} \text {. }
\end{aligned}
$$

FOR THE SECOND CLASS OF THE SECOND CATEGORY.

$$
\begin{aligned}
& \underset{x}{M^{(3}} \underset{y}{M^{0)}}=-\underset{x}{M^{(2}} \underset{y}{M^{1)}}-K_{2} \underset{x}{M^{(1}} \underset{y}{M^{0)}}, \\
& \underset{x}{2 \underset{y}{M^{(3}}{ }_{y}^{1)}}=-\underset{x}{M_{Y}^{2}} \underset{Y}{M^{2}}+K_{4} \underset{x}{M^{0}} \underset{y}{M^{0}}-K_{2} \underset{x}{M} \underset{y}{M}, \\
& \underset{x}{M^{(3}}{\underset{y}{M}}^{2)}=K_{4} \underset{x}{M^{(1}}{\underset{y}{M}}^{0)}, \\
& \underset{x}{M^{3}} \underset{y}{M^{3}}=K_{2} \underset{x}{M^{2}} \underset{y}{M^{2}}+2 K_{4} \underset{x}{M} \underset{y}{(2} M^{0)}+K_{4} \underset{x}{M} \underset{y}{M} .
\end{aligned}
$$

FOR THE SECOND CLASS OF THE FIRST CATEGORY.

$$
\underset{12}{M M_{2}}=K_{2}
$$

Proof OF The Relations in (4.12). The values of index $x$ belong to anyone of the following three groups:

$$
x=a=1,2 ; \quad x=b=3,4 ; \quad x=c=5,6 .
$$


For the values of two different indices $x$ and $y$, we have two cases. The first case is that $x$ and $y$ belong to the same group. The second case is that each of $x$ and $y$ belongs to two different groups. In the first case, it may be easily shown that the relations in (4.12) are identities $0=0$, because in the proof given below each of $A, B, C, D, E$, and $F$ contains the factor $\underset{x}{M}+\underset{y}{M}=0$. Therefore, in the proof of the relations (4.12) it suffices to show the validity of the relations for the second case only. Now let $x, y$, and $z$ be indices each of which belongs to mutually different groups.

In order to prove $(4.12 \mathrm{a})$, consider

$$
A=(\underset{x}{M}+\underset{y}{M})\left(\underset{x}{M^{2}} \underset{y}{M^{2}}+\underset{y}{M^{2}} \underset{z}{M^{2}}+\underset{z}{M^{2}} \underset{x}{M^{2}}\right)
$$

In virtue of (4.6c), we have

$$
A=2 K_{4} \underset{x}{M} \underset{y}{M^{0)}}
$$

On the other hand, the relation (4.6b) gives

$$
\begin{aligned}
& A=\underset{x}{2} M_{y}^{(1} \underset{M^{0)}}{M^{0}}\left[\underset{y}{M^{2}} M^{2}+\underset{y}{M^{2}}\left(-K_{2}-\underset{x}{M^{2}}-\underset{y}{M^{2}}\right)+\underset{x}{M^{2}}\left(-K_{2}-\underset{x}{M^{2}}-\underset{y}{M^{2}}\right)\right]
\end{aligned}
$$

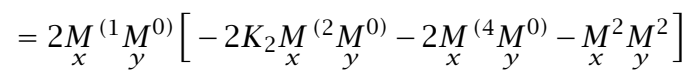

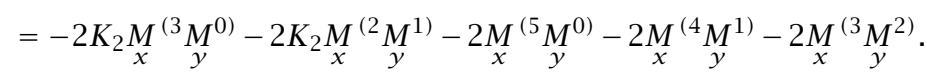

Consequently, the relation (4.12a) follows from (4.15a) and (4.15b).

The remaining relations in (4.12) may be proved similarly by considering

$$
\begin{aligned}
& B=(\underset{x}{M}+\underset{y}{M}) \underset{x}{M_{y}^{(5}}{\underset{y}{0)}}^{0)} \quad \text { for the proof of }(4.12 \mathrm{~b}) \text {, } \\
& C=\underset{x}{M^{2}} M_{y}^{2}(\underset{x}{M}+\underset{y}{M})\left(\underset{x}{M^{2}}+\underset{y}{M^{2}}+\underset{z}{M^{2}}\right), \quad \text { for the proof of }(4.12 \mathrm{c}) \text {, } \\
& D=(\underset{x}{M}+\underset{y}{M}) \underset{x}{M}{ }^{(5} M_{y}^{2)}, \quad \text { for the proof of }(4.12 \mathrm{~d}) \text {, } \\
& E=\underset{x}{M} \underset{y}{M}(\underset{x}{M}+\underset{Y}{M}) \underset{x}{M^{2}} \underset{y}{M^{2}} \underset{z}{M^{2}}, \quad \text { for the proof of (4.12e), } \\
& F=\underset{x}{M} \underset{y}{M}(\underset{x}{M}+\underset{y}{M}) \underset{x}{M}{ }^{(5}{ }_{y}^{2)}, \quad \text { for the proof of (4.12f). }
\end{aligned}
$$

ProOF OF THE RELATIONS IN (4.13) AND (4.14). These relations may be obtained from (4.12) by substituting the corresponding conditions of each case .

Now, we are ready to prove the recurrence relations of the third kind in the following theorem. These relations are very important for the solution of (2.4) or (2.20) in 6- $g$ UFT. We use these relations in our subsequent paper to establish a linear system equivalent to (2.4) and to find a precise and surveyable tensorial representation of 6-dimensional Einstein's connection in terms of the unified field tensor $g_{\lambda \mu}$.

THEOREM 4.5 (The recurrence relations of the third kind in 6-g-UFT). If T is a skewsymmetric tensor in the first two indices, the following recurrence relations hold in 6-g-UFT: 
FOR THE FIRST CLASS .

$$
\begin{aligned}
\stackrel{(50) r}{T} & =-\stackrel{(41) r}{T}-\stackrel{(32) r}{T}-K_{2} \stackrel{(30) r}{T}-K_{2} \stackrel{(21) r}{T}-K_{4} \stackrel{(10) r}{T}, \\
2 \stackrel{(51) r}{T} & =-{ }^{33 r}-2 K_{2} \stackrel{(31) r}{T}-K_{4} T^{11 r}, \\
\stackrel{(52) r}{T} & =-\stackrel{(43) r}{T}-K_{2} \stackrel{(32) r}{T}+K_{6} \stackrel{(10) r}{T} r \\
2 \stackrel{(53) r}{T} & =-\stackrel{44 r}{T}-K_{2}{ }^{33 r}+K_{4} \stackrel{22 r}{T}-2 K_{6} \stackrel{(20) r}{T}+K_{6} \stackrel{11 r}{T}, \\
\stackrel{(54) r}{T} & =K_{4} \stackrel{(32) r}{T}+K_{6} \stackrel{(30) r}{T}+K_{6} \stackrel{(21) r}{T}, \\
& =K_{2} \stackrel{44 r}{T}+2 K_{4} \stackrel{(42) r}{T}+2 K_{6} \stackrel{(40) r}{T}+K_{4} \stackrel{33 r}{T}+2 K_{6} \stackrel{(31) r}{T}+K_{4} \stackrel{22 r}{T} .
\end{aligned}
$$

FOR THE SECOND CLASS WITH THE SECOND CATEGORY.

$$
\begin{aligned}
& \stackrel{(30) r}{T}=-\stackrel{(21) r}{T}-K_{2} \stackrel{(10) r}{T}, \\
& 2 \stackrel{(31) r}{T}=-\stackrel{22 r}{T}-K_{2}{ }^{11 r}+K_{4} \stackrel{00 r}{T}, \\
& \stackrel{(32) r}{T}=K_{4} \stackrel{(10) r}{T}, \\
& T^{33 r}=K_{2} \stackrel{22 r}{T}+2 K_{4} \stackrel{(20) r}{T}+K_{4}{ }^{11 r} T .
\end{aligned}
$$

FOR THE SECOND CLASS WITH THE FIRST CATEGORY.

$$
\stackrel{11 r}{T}=K_{2} \stackrel{00 r}{T} \text {. }
$$

Proof. We first note that the terms in the right-hand side of (3.8a) vanish identically when $x=y$. Therefore, whenever we use (3.8a), it suffices to consider the terms corresponding to the cases $x \neq y$ only. The proof of the above relations follow from (3.8a), using (4.12) for the proof of (4.16), (4.13) for the proof of (4.17), and (4.14) for the proof of (4.18), respectively. For example, the relation (4.16b) may be proved in the following way:

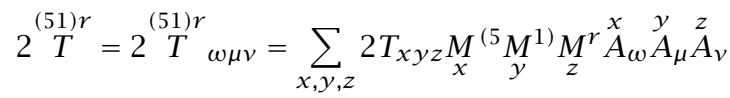

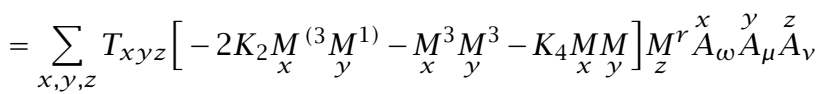

$$
\begin{aligned}
& =-2 K_{2} \stackrel{(31) r}{T}_{\omega \mu \nu}-{ }^{33 r} \omega \mu \nu-K_{4} \stackrel{11 r}{T} \omega \mu \nu \\
& =-2 K_{2} \stackrel{(31) r}{T}-\stackrel{33 r}{T}-K_{4} \stackrel{11 r}{T} \text {. }
\end{aligned}
$$

Acknowledgement. Partially supported by the Basic Science Research Institute Program, Ministry of Education, Republic of Korea, 1997, BSRI-97-1442.

\section{REFERENCES}

[1] K. T. Chung, Einstein's connection in terms of ${ }^{*} g^{\lambda \mu}$, Nuovo Cimento (10) 27 (1963), 12971324. MR 27\#1280. Zbl 115.43605. 
[2] K. T. Chung and K. S. Chang, Degenerate cases of the Einstein's connection in the ${ }^{*} g^{\lambda v}$-unified field theory. I, Tensor (N.S.) 20 (1969), no. 2, 143-149. MR 40\#2320. Zbl 172.28002 .

[3] K. T. Chung and D. H. Cheoi, A study on the relations of two $n$-dimensional unified field theories, Acta Math. Hungar. 45 (1985), no. 1-2, 141-149. MR 86j:83045. Zbl 573.53049.

[4] K. T. Chung and C. H. Cho, Some recurrence relations and Einstein's connection in 2dimensional unified field theory, Acta Math. Hungar. 41 (1983), no. 1-2, 47-52. MR 85d:83061. Zbl 518.53020.

[5] K. T. Chung, P. U. Chung, and I. H. Hwang, The curvature tensors in the Einstein's *gunified field theory II, The contracted SE-curvature tensors of ${ }^{*} g-S E X_{n}$, Bull. Korean Math. Soc. 35 (1998), no. 4, 641-652. Zbl 990.12906.

[6] _ The curvature tensors in the Einstein's * $g$-unified field theory I, The SE-curvature tensors of ${ }^{*} g-S E X_{n}$, J. Korean Math. Soc. 35 (1998), no. 4, 1045-1060.

[7] K. T. Chung and T. S. Han, n-dimensional representations of the unified field tensor ${ }^{*}{ }^{\lambda v}$, Internat. J. Theoret. Phys. 20 (1981), no. 10, 739-747. MR 83m:83049. Zbl 475.53023.

[8] K. T. Chung and I. H. Hwang, Three- and five-dimensional considerations of the geometry of Einstein's ${ }^{*} g$-unified field theory, Internat. J. Theoret. Phys. 27 (1988), no. 9, 11051136. MR 90k:83081. Zbl 652.53058.

[9] K. T. Chung and D. K. Jun, On the Einstein's connection of 3-dimensional unified field theory of the third class, Jour. of NSRI (Yonsei University) 7 (1981), 7-12.

[10] K. T. Chung and S. J. Kang, On the Einstein's connection of 3-dimensional unified field theory of the second class, Jour. of NSRI (Yonsei University) 4 (1979), 5-10.

[11] K. T. Chung and J. W. Lee, On the algebra of 3-dimensional unified field theory of the third class, Jour. of NSRI (Yonsei University) 6 (1980), 1-6.

[12] A. Einstein, The meaning of relativity. 3rd ed., revised, including The Generalized Theory of Gravitation, Princeton University Press, Princeton, NJ, 1950. MR 14,97a. Zbl 050.21208 .

[13] V. Hlavatý, Geometry of Einstein's unified field theory, P. Noordhoff Ltd., Groningen, 1957. MR 20\#5067. Zbl 078.43302.

[14] A. Jakubowicz and J. Klekowska, The necessary and sufficient condition for the existence of the unique connection of the two-dimensional generalized Riemann space, Tensor (N.S.) 20 (1969), 72-74. MR 39\#2088. Zbl 164.52202.

[15] R. S. Mishra, $n$-dimensional considerations of unified theory of relativity. Recurrence relations, Tensor (N.S.) 9 (1959), 217-225. MR 22\#609. Zbl 093.43307.

[16] R. C. Wrede, " $n$ " dimensional considerations of basic principles $A$ and $B$ of the unified theory of relativity, Tensor (N.S.) 8 (1958), 95-122. MR 20\#5073. Zbl 080.21901.

Chung: DePartment of Mathematics, Yonsei University, SeOUl 120-749, Korea

YANG: DEPARTMENT OF MATHEMATICS, KONYANG UNIVERSITY, NONSAN 320-030, KoREA

HWANG: DePARTMENT OF MATHEMATICS, UNIVERSITY OF INCHON, INCHON 402-749, KoREA 


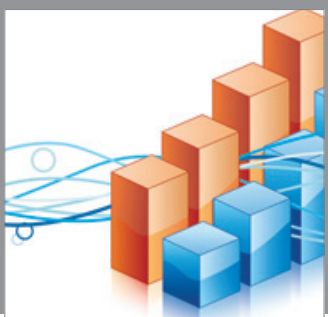

Advances in

Operations Research

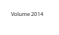

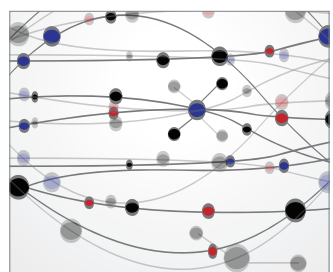

\section{The Scientific} World Journal
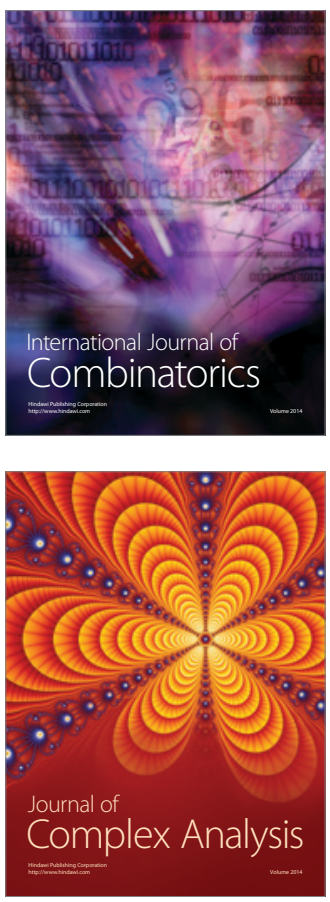

International Journal of

Mathematics and

Mathematical

Sciences
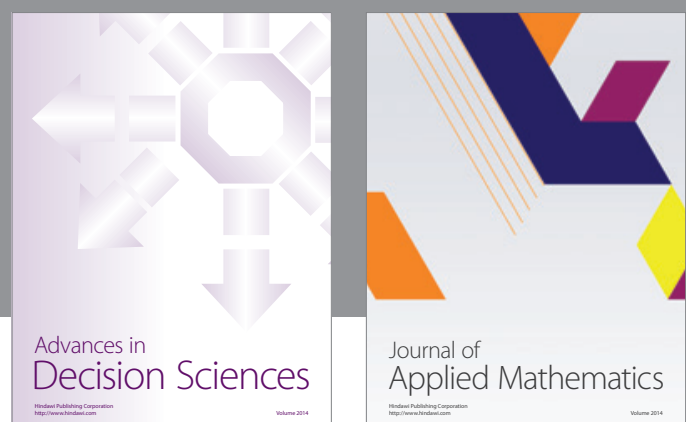

Journal of

Applied Mathematics
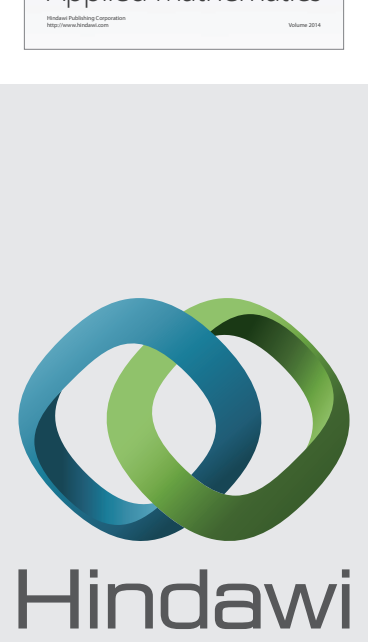

Submit your manuscripts at http://www.hindawi.com
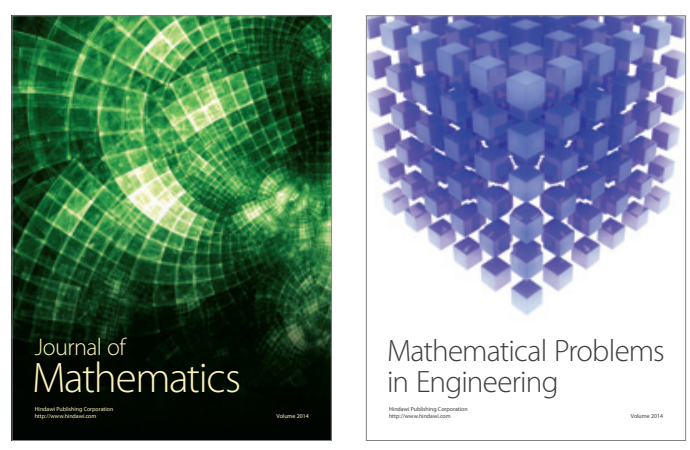

Mathematical Problems in Engineering
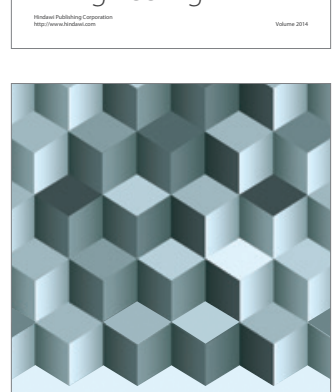

Journal of

Function Spaces
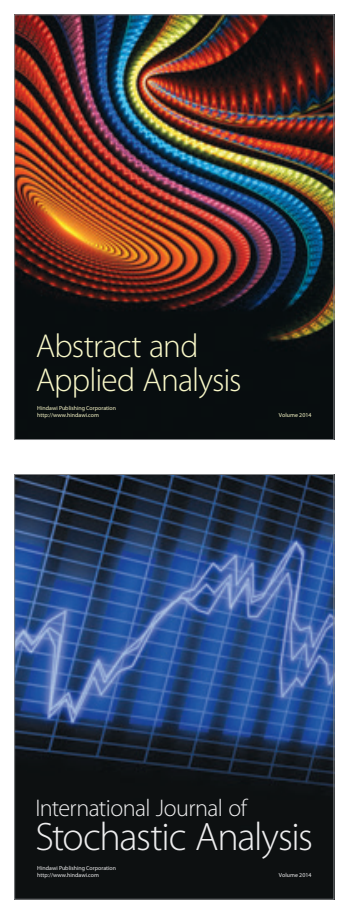

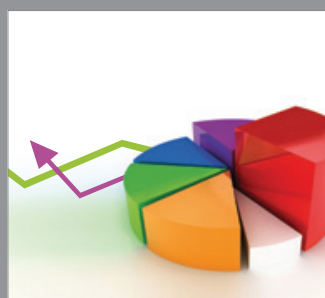

ournal of

Probability and Statistics

Promensencen
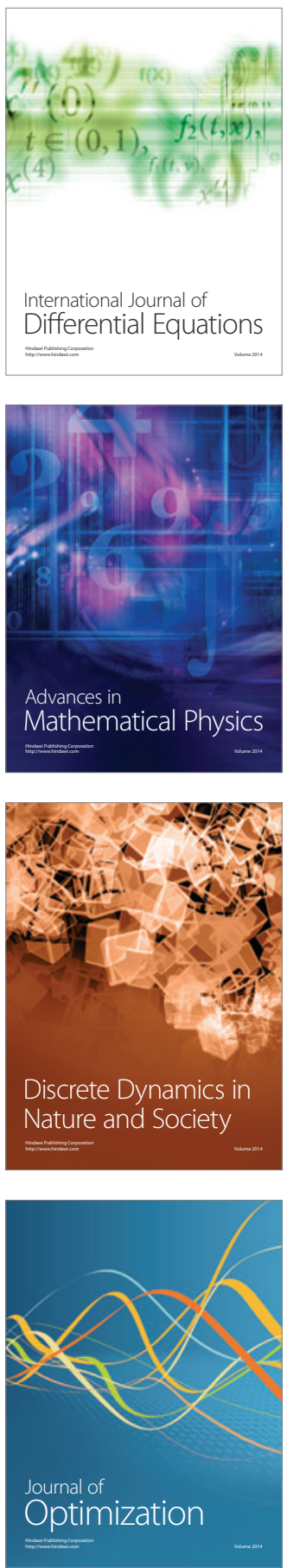